\title{
Anti-plane crack in human bone. I. Mathematical modelling
}

\author{
E. M. Craciun ${ }^{1 *}$, M. Marin ${ }^{2}$, A. Rabaea ${ }^{3}$
}

\begin{abstract}
We consider an anti-plane crack in a bone, considered as an initially deformed orthotropic, linear elastic composite material. Elastic incremental fields in the composite material are obtained following theories of Guz's representation and of Riemann-Hilbert problem. Critical values of crack propagation angle are determined using Sih's generalized criterion.
\end{abstract}

\section{Introduction}

Although the plane modes of fracture (Mode I, Mode II and mixed mode I+II) have been the most studied human bone fracture, bones are loaded in antiplane mode, also. Many cracks formed in bone are loaded in anti-plane Mode III of fracture due to the hierarchical and directional nature of its structure. Bone is a composite of collagen and hydroxypathite (HA) which includes twisted peptide chains at nanoscale, HA-impregnated twisted collagen fibers and osteon structures at the scale of several hundred micrometer in size. Many factors affect the fracture toughness of the human bones such as ageing, diseases, environmental conditions, and so on. Human bone is often considered to be orthotropic composite material with organized microstructure; the structural axes of orthotropic symmetry being defined by the bone microstructure, [5], [17]-[18].

Key Words: Mode III of fracture, initial fields, Riemann-Hilbert problem, Femur and Tibia bones

Received: : 15.03 .2017

Revised: 15.06.2017

Accepted: 25.06.2017 
Few mathematical models were developed for fracture of bone as a prestressed composite material. Consequently, in this work we study a $2 \mathrm{D}$ quasistatic anti-plane crack propagation in a pre-stressed human bone, regarded as an homogeneous elastic composite. We start our study with general theory of incremental fields of initially loaded elastic composites. Many analytical and numerical studies were done in this direction of obtaining the incremental behaviour of cracked elastic composites, [1], [9]-[11]. From the literature, [2]-[4], [12]-[15], we know that in the case of cracked pre-stressed bodies, the incremental stress and displacements fields are described using complex potentials and the initial stress, and play an important role to determine crack propagation. Our second aim is to generalize Sih's fracture energy density criterion (SED) [16] to determine crack propagation direction in initially loaded human bones with anti-plane cracks. For doing this we performed an asymptotical analysis, to find the complex potentials, incremental stress and displacement fields. We consider as examples two configurations of anti-plane crack in prestressed Femur and Tibia human bones. To obtain crack propagation angle we determined the minimum of strain-energy-density versus initial stress and polar angle. We found that anti-plane crack in pre-stressed Femur and Tibia human bone is propagating along or perpendicular to the crack line depending by the elastic constants of human bone and by the initial applied field.

\section{Mathematical modeling of the anti-plane crack}

We suppose that the bone, regarded as an initial deformed orthotropic material, contains a crack of length $2 a>0$ situated on the $x_{1}$ axis and has infinite extent in the direction of the $x_{3}$ axis. As usual in continuum mechanics, the crack is represented as a cut with two faces. We assume that the initial deformed equilibrium configuration of the body is homogeneous and locally stable and that at large distances from the crack the incremental stress and displacements fields vanish.

We have the following boundary conditions on the crack faces:

$$
\vartheta_{23}\left(x_{1}, 0^{+}\right)=\vartheta_{23}\left(x_{1}, 0^{-}\right)=-\tau\left(x_{1}\right) \text { for }\left|x_{1}\right|<a,
$$

$\vartheta_{23}$ is the involved component of the incremental nominal stress $\boldsymbol{\vartheta}$ and $\tau=$ $\tau\left(x_{1}\right)$ being the given value of the incremental anti-plane tangential surface force.

Using representation formulae [6]-[8], [12] we have:

$$
\begin{aligned}
& \vartheta_{23}=-2 \operatorname{Re} \Phi_{3}^{\prime}\left(z_{3}\right), \\
& \vartheta_{13}=2 \operatorname{Re} \mu_{3} \Phi_{3}^{\prime}\left(z_{3}\right),
\end{aligned}
$$




$$
u_{3}=-2 \omega_{2332}^{-1} \operatorname{Re} \mu_{3}^{-1} \Phi_{3}\left(z_{3}\right) .
$$

The above relations, due to Guz [12], express the incremental fields corresponding to the anti-plane state, by a single complex potential $\Phi_{3}=\Phi_{3}\left(z_{3}\right)$ depending on the complex variable $z_{3}=x_{1}+\mu_{3} x_{2}, \mu_{3}$ being the root of the characteristic equation of equilibrium having the value [8]

$$
\mu_{3}=i \sqrt{\frac{\omega_{1331}}{\omega_{2332}}} .
$$

In Eq. (3) the instantaneous elasticities $\omega_{1331}, \omega_{2332}$ are given by shear moduli $G_{13}, G_{23}$ and the initial stress $\sigma$ as follows:

$$
\omega_{1331}=G_{13}+\sigma, \omega_{2332}=G_{23} .
$$

The initial stress $\sigma$ represents a pre-stress when it modulus is negative and a tensile stress when it modulus is positive.

We introduce the potential

$$
\Psi\left(z_{3}\right)=\Phi^{\prime}\left(z_{3}\right)
$$

Using Eqs. (2.1) and (2.2) we get the following Riemann-Hilbert problems:

$$
\begin{aligned}
& (\Psi+\bar{\Psi})^{+}\left(x_{1}\right)+(\Psi+\bar{\Psi})^{-}\left(x_{1}\right)=2 \tau\left(x_{1}\right), \\
& (\Psi-\bar{\Psi})^{+}\left(x_{1}\right)-(\Psi-\bar{\Psi})^{-}\left(x_{1}\right)=0,\left|x_{1}\right|<a .
\end{aligned}
$$

Following Muskhelishvili's formalism [13] we get solution of our RiemannHilbert problems:

$$
\Psi\left(z_{3}\right)=\frac{1}{2 \pi \sqrt{z_{3}^{2}-a^{2}}} \int_{-a}^{a} \frac{\tau(t) \sqrt{a^{2}-t^{2}}}{t-z_{3}} d t .
$$

From Eqs. (2), (3), (7) and taking into account that the incremental displacement $u_{3}$ vanishes at large distances from the crack, we obtain the following important result, due to symmetry of the material and to applied stress fields, that the incremental displacement is vanishing on the crack line. In a small vicinity of the crack tip $a$ we have:

$$
x_{1}=a+r \cos \varphi, x_{2}=r \sin \varphi, z_{3} \approx a, \sqrt{z_{3}^{2}-a^{2}}=\sqrt{2 a r} \Xi(\varphi),
$$

where $\Xi(\varphi)=\sqrt{\cos \varphi+\mu_{3} \sin \varphi}$. 
Using Eqs. (2), (7) and (8) we get the following asymptotic expressions of the complex potentials and incremental fields:

$$
\begin{gathered}
\Psi\left(z_{3}\right)=-\frac{K}{2 \sqrt{2 \pi r}}(\Xi(\varphi))^{-1}, \Phi\left(z_{3}\right)=-K \sqrt{\frac{r}{2 \pi}} \Xi(\varphi), \\
\vartheta_{23}=\frac{K}{\sqrt{2 \pi r}} \operatorname{Re}[\Xi(\varphi)]^{-1}, \vartheta_{13}=-\frac{K}{\sqrt{2 \pi r}} \operatorname{Re}\left[(\Xi(\varphi))^{-1} \mu_{3}\right], \\
u_{3}=\frac{2}{\omega_{2332}} \sqrt{\frac{r}{2 \pi}} K \operatorname{Re}\left[\Xi(\varphi) \mu_{3}^{-1}\right],
\end{gathered}
$$

where $K$ represents the stress intensity factor corresponding to Mode III of fracture, [7]-[8],

$$
K=\frac{1}{\sqrt{\pi a}} \int_{-a}^{a} \tau(t) \sqrt{\frac{a+t}{a-t}} d t .
$$

In what follows we assume that $\tau\left(x_{1}\right)=\tau=$ const. for $\left|x_{1}\right|<a$.

From (12), we get:

$$
K=\tau \sqrt{\pi a}
$$

and Eqs. (9)-(11) become:

$$
\begin{gathered}
\vartheta_{23}=\tau \sqrt{\frac{a}{2 r}} \operatorname{Re}[\Xi(\varphi)]^{-1}, \vartheta_{13}=-\tau \sqrt{\frac{a}{2 r}} \operatorname{Re}\left[(\Xi(\varphi))^{-1} \mu_{3}\right], \\
u_{3}=\tau \frac{\sqrt{2 r a}}{\omega_{2332}} \operatorname{Re}\left[\Xi(\varphi) \mu_{3}^{-1}\right] .
\end{gathered}
$$

\section{Generalized strain energy density fracture criterion}

Let be $W$ the involved strain energy density, [16]

$$
\frac{d W}{d V}=\frac{1}{2}\left(\vartheta_{13} u_{3,1}+\vartheta_{23} u_{3,2}\right) .
$$

Using Eqs. (14)-(16) we get the following value for $W$ :

$$
\frac{d W}{d V}=\frac{(|\Xi(\varphi)|)^{2}}{\omega_{2332}} .
$$

Sih's generalized fracture energy density criterion (SED) [16] is applied to all composite materials, loading types and structure geometries, with or without initial defects. The strain energy density function $\frac{d W}{d V}$ will be assumed to have the form: 


$$
\frac{d W}{d V}=\frac{S}{r} .
$$

The fundamental quantity is the strain energy density factor $S$, the amplitude of the energy field that possesses an $r^{-1}$-type of singularity. We consider the situation of brittle crack growth when crack initiation coincides with final instability. The factor $S$ defined as in Eq. (18), represents the local energy release for a segment of crack growth $r$.

Sih's new fracture criterion is based on the following two hypothesis, [16]:

H1: Crack will start in a radial direction $\varphi_{c}$ along which the intensity $S(\varphi)$ of the strain-energy-density field has a minimum.

H2: The critical intensity $S_{c}$ governs the onset of crack propagation and represents a material constant independent of the crack geometry and loading. Here, $S_{c}$ is a material parameter

$$
S\left(\varphi_{c}\right)=S_{c}
$$

From Eqs. (17) and (18) we get:

$$
S(\varphi)=\frac{K^{2} a}{4 \omega_{2332}}\left(\cos ^{2} \varphi+q^{2} \sin ^{2} \varphi\right)^{-1 / 2} .
$$

\section{Crack propagation angle in Femur and Tibia Bone}

The present 2D quasistatic mathematical model provides a means to find crack propagation angle of an anti-plane crack in a human bone regarded as an anisotropic material with initial fields.

We consider two numerical examples:

- A. Anti-plane crack in Femur bone and

- B. Anti-plane crack in Tibia bone.

Femur bone is characterized by the following engineering constants (values in GPa), [5]:

$E_{1}=12.0 ; E_{2}=13.4 ; E_{3}=20.0 ; G_{12}=4.53, G_{13}=5.61, G_{23}=6.23, \nu_{12}=$ $0.37 ; \nu_{13}=0.22 ; \nu_{23}=0.23 ; \nu_{21}=0.42 ; \nu_{31}=0.37 ; \nu_{32}=0.35$.

Tibia bone is characterized by the following engineering constants (values in GPa), [5]:

$E_{1}=6.91 ; E_{2}=8.51 ; E_{3}=18.4 ; G_{12}=2.41, G_{13}=3.56, G_{23}=4.91, \nu_{12}=$ $0.49 ; \nu_{13}=0.12 ; \nu_{23}=0.14 ; \nu_{21}=0.62 ; \nu_{31}=0.32 ; \nu_{32}=0.31$.

We consider that the modulus of our initial stress is $|\sigma|<1.5 \mathrm{GPa}$. 
Let be $w$ normalized incremental strain energy density and $t_{23}$ normalized incremental shear stress, $i e$.

$$
w=S(\varphi) \frac{4 \omega_{2332}}{\pi \tau^{2} a^{2}}, t_{23}=\vartheta_{23} \tau \sqrt{\frac{2 r}{a}} .
$$

Using Eq. (21) and Sih's new fracture criterion we observe that crack will start to propagate if the normalized strain-energy-density $w$ has a minimum.
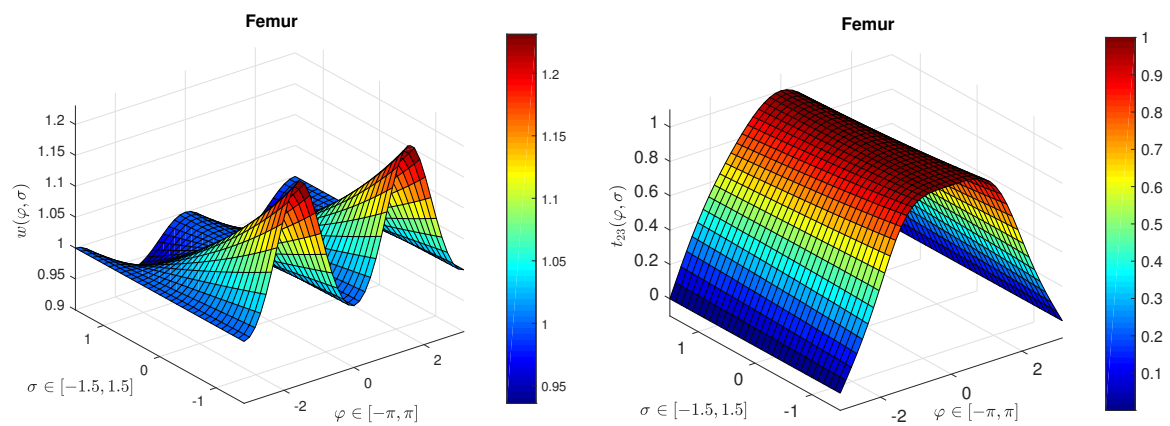

Figure 1: Femur bone: Normalized incremental strain energy density $w$ and normalized incremental shear stress $t_{23}$ versus $\varphi$ and $\sigma$
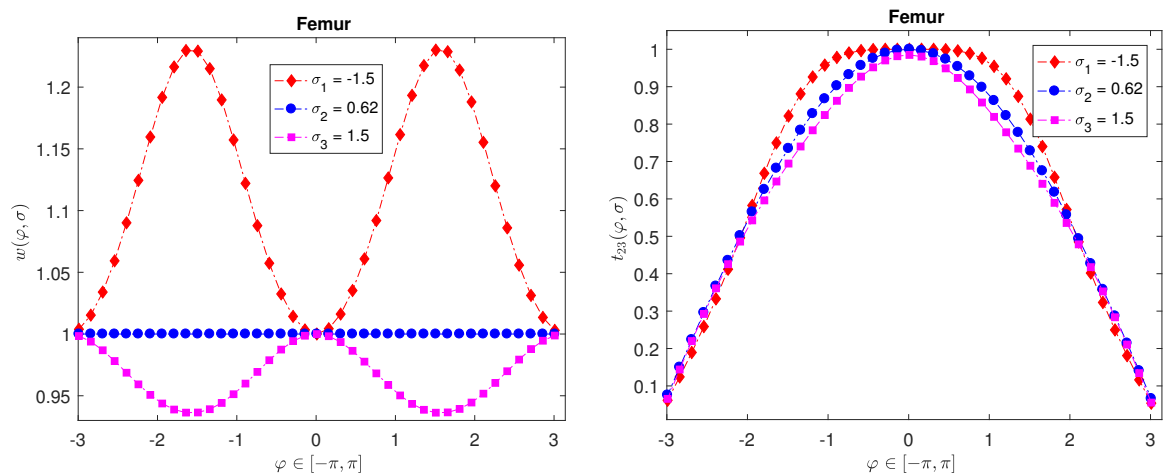

Figure 2: Femur bone: Normalized incremental strain energy density $w$ and normalized incremental shear stress $t_{23}$ versus $\varphi$ for $\sigma \in\{-1.5,0.62,1,5\}$

In Figs. 1 and 3 (left) we plotted the normalized incremental strain energy density $w$ versus $\varphi$ and $\sigma$ for Femur and Tibia bones. We observe that the 
minimum of $w$ is related versus $\sigma$ and is in the vicinity of $0^{\circ}$ and in the vicinity of $\pm 90^{\circ}$.
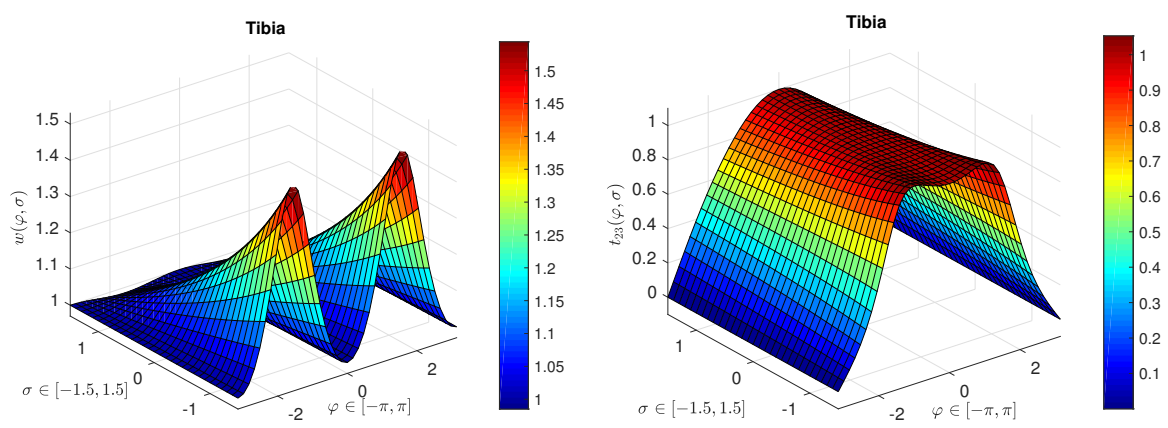

Figure 3: Tibia bone: Normalized incremental strain energy density $w$ and normalized incremental shear stress $t_{23}$ versus $\varphi$ and $\sigma$
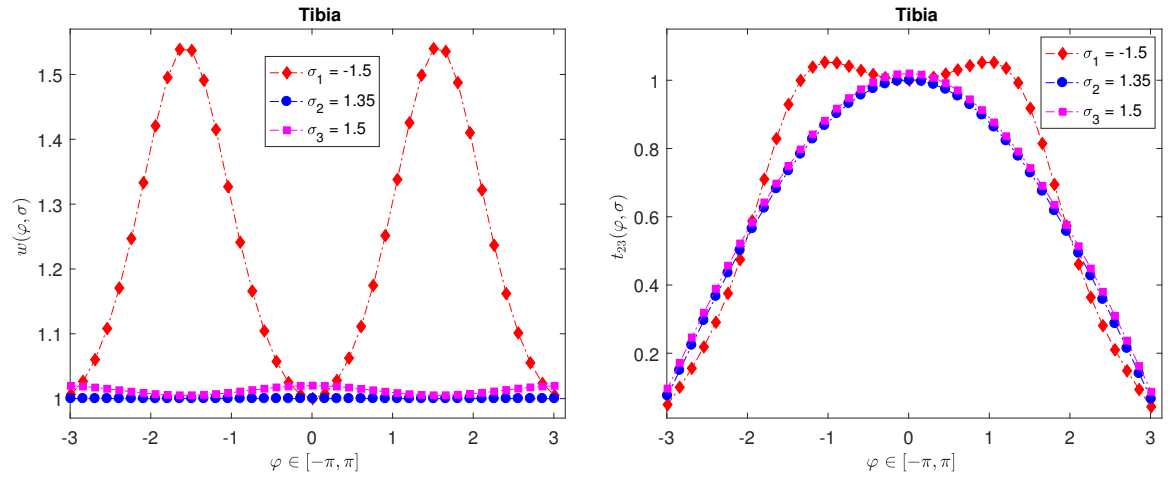

Figure 4: Tibia bone: Normalized incremental strain energy density $w$ and normalized incremental shear stress $t_{23}$ versus $\varphi$ versus $\varphi$ for $\sigma \in\{-1.5,1.35,1,5\}$

In Figs. 2 and 4 (left) we plotted the normalized incremental strain energy density $w$ versus $\varphi$ for three values of $\sigma, \sigma \in\{-1.5,0.62,1,5\}$ for Femur bone and $\sigma \in\{-1.5,1.35,1,5\}$ for Tibia bone, respectively.

Taking into account that the derivative $w^{\prime}$ of the normalized incremental strain energy density function $w$ versus $\varphi$ is

$$
w^{\prime}=-\frac{\left(q^{2}-1\right) \sin 2 \varphi}{2}\left(\cos ^{2} \varphi+q^{2} \sin ^{2} \varphi\right)^{-3 / 2}
$$


we conclude that minimum of the normalized incremental strain energy density $w$ versus $q$ is obtained in the vicinity of $0^{\circ}$ for $\sigma<0.62$, (respectively $\sigma<1.35$ ) and is obtained in the vicinity of $\pm 90^{\circ}$ for $\sigma>0.62$, (respectively, $\sigma>1.35$ ) for Femur, (respectively, for Tibia bone).

When $\sigma=0.62$ and $\sigma=1.35$ for Femur and Tibia bone, respectively we have $q=w=1$ and Sih's new fracture criterion could not be applied.

In Figs. 1 and 3 (right) we plotted the normalized incremental shear stress $t_{23}$ versus $\varphi$ and $\sigma$ for Femur and Tibia bones.

From Figs. 2 and 4 (left), where we plotted the normalized incremental shear stress $t_{23}$ versus $\varphi$ for three values of $\sigma, \sigma \in\{-1.5,0.62,1,5\}$ for Femur bone and $\sigma \in\{-1.5,1.35,1,5\}$ respectively, for Tibia bone. We remark that for the case of pre-stress the minimum of incremental shear stress $t_{23}$ is obtained in the vicinity of $0^{\circ}$, also. In this case we can conclude that could exist connection between Sih's new fracture criterion and the minimum of incremental shear stress $\vartheta_{23}$. This represents an important observation, due to the fact that the minimum of incremental shear stress $\vartheta_{23}$ could be determined by experimental tests using testing machines.

\section{Conclusions}

We consider an anti-plane crack in a bone, considered as an initially deformed orthotropic, linear elastic composite material. The anti-plane state produced in the body is determined using the theories of Guz' s representation and of Riemann-Hilbert problem, using complex potentials.

The presented 2D quasistatic mathematical model provides a means to find crack propagation angle of an anti-plane crack in a human bones regarded as anisotropic materials with initial fields. We observed in two cases of an anti-plane crack in Femur and Tibia bones, regarded as initially deformed orthotropic material that the crack will propagate along or perpendicular its line, depending by the initial applied stress field and by the elastic constants. Also, in the case of pre-stress, we observed that a connection between Sih's new fracture criterion and the minimum of incremental shear stress exists, both indicates that the minimum is in the vicinity of $0^{\circ}$. This fact must be confirmed by future anti-plane fracture tests.

\section{References}

[1] Adamus, J., Lacki, P., Numerical simulation of forming titanium drawn part, 51(2)(2016), pp. 391-400

[2] Argani, L., Bigoni, D., Capuani, D. and Movchan, N.V., Cones of localized shear strain in incompressible elasticity with prestress: Green's function and integral representations, Proc. R. Soc. A, 470(2014), pp. 01-17 
[3] Benvenuti, E., A regularized XFEM framework for embedded cohesive interfaces, Comput. Methods Appl. Mech. Engrg. 197(2008) pp. 43674378

[4] Capuani, D., Bigoni, D. and Brun, M., Integral representations at the boundary for Stokes flow and related symmetric Galerkin formulation, Arch. Mech., 2005, 57(5)(2005), pp. 363-385

[5] Cowin, S.C. et all, Properties of bone in Handbook of Bioengineering, McGraw-Hill, New York, 1987

[6] Craciun, E.M., Soos, E., Interaction of two unequal cracks in a prestressed fiber reinforced composite, Int. J. of Fracture 94(1998), pp. 137-159

[7] Craciun, E.M., Soos, E., Crack propagation in the third fracture mode, Rev. Roum. Math. Pures Appl. 45(2000), pp. 229-234

[8] Cristescu, N., Craciun, E.M. and Soos, E., Mechanics of Elastic Composites, CRC Press, Chapman \& Hall, 2004

[9] Ghita, C., Pop, N. and Popescu, I. N., Existence result of an effective stress for an isotropic visco-plastic composite, Computational Materials Science, 64(2012), pp. 52-56 .

[10] Gourgiotis, P.A., Bigoni, D., Stress channelling in extreme couple-stress materials Part I: Strong ellipticity, wave propagation, ellipticity, and discontinuity relations, Journal of the Mechanics and Physics of Solids, 88(2016), pp. 1501668

[11] Gourgiotis, P.A., Bigoni, D., Stress channelling in extreme couple-stress materials Part II: Localized folding vs faulting of a continuum in single and cross geometries, Journal of the Mechanics and Physics of Solids, 88(2016), pp. 169-185

[12] Guz, A.N., Mechanics of brittle fracture of pre-stressed materials, Visha Shcola, Kiev, 1983

[13] Muskhelishvili, N.I., Some Basic Problems of Mathematical Theory of Elasticity, Nordhoff, Groningen, 1953

[14] Radi, E., Bigoni, D. and Capuani, D., Effects of pre-stress on crack-tip fields in elastic, incompressible solids, International Journal of Solids and Structures, 39(2002), pp. 3971-3996

[15] Radi, E., On the effects of characteristic lengths in bending and torsion on Mode III crack in couple stress elasticity, International Journal of Solids and Structures, 45(2008), pp. 3033-3058

[16] Sih, G.C. A special theory of crack propagation, in: Mechanics of Fracture, vol. I, Editor G.C. Sih, Noordhoof Int. Publ. Leyden, 1973, pp. XXI-XLV

[17] Taylor, W.R., Determination of orthotropic bone elastic constants using FEA and modal analysis, Journal of Biomechanics 35 (2002), pp. 767-773

[18] Zimmermann E.A. et all, Mixed-mode fracture of human cortical bone, Biomaterials, 30 (2009) pp. 58775884. 
Eduard Marius CRĂCIUN,

Faculty of Mechanical, Industrial and Maritime Engineering,

Ovidius University of Constanta,

900527 Constanta, Romania,

Email: mcraciun@univ-ovidius.ro

Marin MARIN,

Department of Mathematics and Computer Science,

Transilvania University of Brasov,

500188 Brasov, Romania,

Email: m.marin@unitbv.ro

Adrian RĂBÂEA

Department of Mathematics and Informatics

Technical University of Cluj-Napoca, N.U.C.B.M.

430122 Baia Mare, Romania

Email: adrian1803@gmail.com 